\title{
Output control of a single-airscrew helicopter's longitudinal motion spectrum
}

\begin{abstract}
The problem of stabilization law synthesis of a single-airscrew helicopter's longitudinal motion for lack of information about the vertical speed of its motion is analytically solved. The solution is based on the method of output control synthesis of the MIMO-system motion spectrum to be used as the basis of an especially designed multilevel decomposition of the dynamic system model in state space.
\end{abstract}

Keywords: single-airscrew helicopter, longitudinal motion, linear MIMO-system, stabilization, output feedback, motion spectrum, Control laws synthesis, multilevel decomposition
Volume 4 Issue 3 - 2018

\author{
Nikolay E Zubov, Vladimir N Ryabchenko, \\ Igor V Sorokin
}

Moscow, Bauman Moscow State Technical University, Russia

Correspondence: Nikolay E Zubov, Moscow, Bauman Moscow State Technical University, Russia, Tel +79637637687, Email Nikolay.Zubov@rsce.ru

Received: August 17,2017| Published: May 07, 2018

\section{Introduction}

The physical principle of a single-airscrew helicopter's spatial motion provides the opportunity to categorize it as a complex multidimensional dynamic system (control object). At the same time, it is possible to mark out the following problems in less detail, which can be attributed to the control of helicopters as multidimensional dynamic systems. ${ }^{1,2}$

a. The problem of stabilization, or the problem of stabilizing control law synthesis, i.e. determination of a feedback (of a controller), which provides stability for a disturbed motion. In searching for this kind of problem solution, as a rule, modal control methods or pole control methods are used..$^{3-6}$

b. The closed-loop system decoupling problem, i.e. determination of a controller's coefficients that provide decoupling of the control object's subsystems. The group of methods used is based on the analysis and usage of the reference system operators' kernels. ${ }^{7,8}$

c. The problem of making a set of stabilizing control laws, i.e. obtaining all, or almost all, control laws (algorithms), which provide stability for the closed-loop system, if the solution to the first problem, from those listed above, is not the only one. As a rule, the methods used are based on the solutions to Diophantine equations and the subsequent parameterization (for instance, the Youla-Kucera parametrization). ${ }^{7,9-12}$

It is possible to find other, not so commonly used, statements and solutions to the problem. For instance, there is a statement and a solution to the problem when stability and decoupling of the closed-loop controlled system, and also specified placement of the invariant system's zeros, i.e. complex frequencies, on which the closed-loop system "locks" completely or partially, can be provided simultaneously. The strengthened type of decoupling, when it is necessary to provide not only the block-diagonal form of the closedloop system's operator, but also specific placement of the invariant system's zeros on the complex plane, belongs to it. Many of the practically important solutions to the problems listed above are not accompanied by published methods on how to obtain them. The analytical solutions have to fill this gap. In this paper for the first time the analytical solution to the problem of a single-airscrew helicopter's stabilization in the vertical plane with the specified placement of poles and the incomplete measurement vector of motion parameters is presented. We use the linearized model of longitudinal motion of a single-airscrew helicopter $(\mathrm{SH})$ having the following form: $:^{13}$

$$
\left(\begin{array}{c}
\dot{V}_{x} \\
\dot{V}_{y} \\
\dot{\omega}_{z} \\
\dot{v}
\end{array}\right)=\left(\begin{array}{cccc}
a_{V_{x}}^{V_{x}} & a_{V_{x}}^{V_{y}} & a_{V_{x}}^{\omega_{z}} & a_{V_{x}}^{v} \\
a_{V_{y}}^{V_{x}} & a_{V_{y}}^{V_{y}} & a_{V_{y}}^{\omega_{z}} & a_{V_{y}}^{v} \\
a_{\omega_{z}} & a_{\omega_{z}}^{V_{y}} & a_{\omega_{z}}^{\omega_{z}} & 0 \\
0 & 0 & 1 & 0
\end{array}\right)\left(\begin{array}{c}
V_{x} \\
V_{y} \\
\omega_{z} \\
v
\end{array}\right)+\left(\begin{array}{cc}
b_{V_{x}}^{u_{x}} & b_{V_{x}}^{u_{\mathrm{gp}}} \\
b_{V_{y}}^{u_{x}} & b_{V_{y}}^{u_{\mathrm{gp}}} \\
b_{\omega_{z}}^{u_{x}} & b_{\omega_{z}}^{u_{\mathrm{gp}}} \\
0 & 0
\end{array}\right)\left(\begin{array}{c}
u_{x} \\
u_{\mathrm{gp}}
\end{array}\right) \cdot(1)
$$

Here $V_{x}$ - deviation from specified value of the longitudinal speed; $V \stackrel{x}{-}$-deviation from specified value of the vertical speed; $v$ - deviation from specified value of the pitch angular velocity; $v-$ deviation from specified value of the angle of pitch; $u_{x}$ - deviation angle of a main rotor's cone in the longitudinal direction; $a_{V_{x}}^{V_{x}}-$ general pitch of a main rotor; $a_{V_{x}}^{V_{x}}, a_{V_{x}}^{V_{y}}, a_{V_{x}}^{\iota_{z}}, a_{V_{y}}^{V_{x}}, a_{V_{y}}^{V_{x}},{ }_{V_{V_{x}}}^{\tilde{o}}$, $a_{V_{y}}^{\tilde{g}}, a_{V_{y}}^{\tilde{o}}, a_{\dot{u}_{z}}^{V_{x}}, a_{\grave{u}_{z}}^{\grave{u}_{z}}, a_{\dot{u}_{z}}^{\grave{u}_{z}}, b_{V_{x}}^{u_{x}}, b_{V_{y}}^{u_{x}}, b_{V_{y}}^{u_{x}}, b_{V_{y}}^{u_{\mathrm{gp}}}, b_{\dot{u}_{z}}^{u_{x}}, b_{\dot{u}_{z}}^{u_{\mathrm{gp}}^{u}}$ - linearization coefficients. ${ }^{8,14}$

We use the following notation

$$
x=\left(\begin{array}{llll}
\Delta V_{x} & \Delta V_{y} & \Delta \omega_{z} & \Delta v
\end{array}\right)^{T}, u=\left(\begin{array}{ll}
\Delta u_{x} & \Delta u_{g p}
\end{array}\right)^{T},
$$

and also

$$
\begin{aligned}
& a_{11}=a_{V_{x}}^{V_{x}}, a_{12}=a_{V_{x}}^{V_{y}}, a_{13}=a_{V_{x}}^{\grave{u}_{z}}, a_{14}=a_{V_{x}}^{\tilde{o}}, \\
& a_{21}=a_{V_{y}}^{V_{x}}, a_{22}=a_{V_{y}}^{V_{y}}, a_{23}=a_{V_{y}}^{\grave{u}_{z}}, a_{24}=a_{V_{y}}^{\tilde{o}}, \\
& a_{31}=a_{\grave{u}_{z}}^{V_{x}}, a_{32}=a_{\dot{u}_{z}}^{V_{y}}, a_{33}=a_{\dot{u}_{z}}^{u_{z}}, \\
& b_{21}=b_{V_{y}}^{u_{x}}, b_{22}=b_{V_{y}}^{u_{\mathrm{gp}}}, \\
& b_{21}=b_{V_{y}}^{u_{x}}, b_{22}=b_{V_{y}}^{u_{\mathrm{gp}}}, \\
& b_{31}=b_{\omega_{z}}^{u_{x}}, b_{32}=b_{\omega_{z}}^{u \mathrm{gp}},
\end{aligned}
$$


Then instead of expression (1) we will obtain the following linearized model of the SH longitudinal motion:

$$
\dot{\mathrm{x}}=\left(\begin{array}{cccc}
a_{11} & a_{12} & a_{13} & a_{14} \\
a_{21} & a_{22} & a_{23} & a_{24} \\
a_{31} & a_{32} & a_{33} & 0 \\
0 & 0 & 1 & 0
\end{array}\right) \mathrm{x}+\left(\begin{array}{cc}
b_{11} & b_{12} \\
b_{21} & b_{22} \\
b_{31} & b_{32} \\
0 & 0
\end{array}\right) \mathrm{u}
$$

Here after it will be considered that information about vertical speed of the SH motion is not available. As a result the system output vector can be expressed as

$$
\mathrm{y}=\left(\begin{array}{lll}
\Delta V_{x} & \Delta \omega_{z} & \Delta v
\end{array}\right)^{\mathrm{T}} .
$$

Taking into consideration the assumptions made, the initial mathematical model can be written in the form of a dynamic MIMOsystem of the "input - state - output" type:

$$
\dot{\mathrm{x}}=\mathrm{Ax}+\mathrm{Bu}, \quad \mathrm{y}=\mathrm{Cx},
$$

Where $\mathrm{x} \in \mathbb{R}^{n}, \quad n=4$ is the state vector; $\mathrm{u} \in \mathbb{R}^{r}$ is the input vector (control vector), where $r=2 ; \mathbf{y} \in \mathbb{R}^{m}$ is the output vector (measurement vector), where $m=3$. Here $\mathbb{R}$ is the set of real numbers.

In the equations (3) the corresponding matrices can be written as

$$
A=\left(\begin{array}{cccc}
a_{11} & a_{12} & a_{13} & a_{14} \\
a_{21} & a_{22} & a_{14} & a_{24} \\
a_{31} & a_{32} & a_{33} & 0 \\
0 & 0 & 1 & 0
\end{array}\right), B=\left(\begin{array}{cc}
b_{11} & b_{12} \\
b_{21} & b_{22} \\
b_{31} & b_{32} \\
0 & 0
\end{array}\right), \quad C=\left(\begin{array}{cccc}
1 & 0 & 0 & 0 \\
0 & 0 & 1 & 0 \\
0 & 0 & 0 & 1
\end{array}\right) \text {. }
$$

If, as a control law(3), to suggest the expression of the following form:

$$
\mathrm{u}=\mathrm{Fy}=\mathrm{FCx},
$$

Where $F \in \mathbb{R}^{r \times m}$ is a matrix of the output controller, then, in accordance with ${ }^{2}$ for the system under consideration (3) - (5) a case of the dynamic MIMO-system output control will take place.

Here after we assume that matrix $B \in \mathbb{R}^{4 \times 2}$ (4) has a full rank ( rank $\mathrm{B}=2$ in this case), or that its equivalent matrix $B^{T} B$ is invertible, i.e. $\operatorname{det}\left(B^{\mathrm{T}} B\right) \neq 0$. Let us now consider the matrix spectrum $A \in \mathbb{R}^{4 \times 4}$ (5). It will be understood as a set of matrix $A$ eigen values (poles), $\operatorname{eig}(A)=\left\{\lambda_{i} \in \mathbb{C}: \operatorname{det}\left(\lambda_{i} I_{4}-A\right)=0, i=\overline{1,4}\right\}$. Here $\mathrm{I}_{4}-$ identity matrix of size $4 \times 4, \mathbb{C}-$ the set of complex numbers. Let $\Lambda$ be the given spectrum of the matrix $\mathrm{A}+\mathrm{BFC}$ of the corresponding close-loop controlled system, i.e.

$$
\Lambda=\left\{\hat{\lambda}_{1}, \hat{\lambda}_{2}, \hat{\lambda}_{3}, \hat{\lambda}_{4}\right\} .
$$

It is required to determine (i.e. synthesize) explicitly the controller matrix FÎR ${ }^{2 \times 3}$, such that the equality $\Lambda=\operatorname{eig}(A+B F C)$ should be satisfied exactly. The complexity of this problem is a necessity for obtaining a solution in explicit analytical form, since $m \geq r$ matrices in (2) have a piecewise constant form. This paper is dedicated to solving of the problem.

\section{Decomposition of a dynamic system}

As a first step of the given problem solution we will consider the multilevel decomposition of the SH model suggested. ${ }^{15-17}$ Since in this case the inequality $m \geq r$ (i.e. the number of system's outputs is greater than the number of its inputs) is implemented, then, in general, not taking into consideration specific numerical values for $m$ and $r$, we consider the multilevel decomposition of system (3) - (5) of the following form: -zero decomposition level

$$
\mathrm{A}_{0}=\mathrm{A}, \quad \mathrm{B}_{0}=\mathrm{B}, \quad \mathrm{C}_{0}=\mathrm{C}
$$

$-k$ th decomposition level $(k=\overline{1, M}$, where $M=\operatorname{ceil}(n / r)$, -is the operation of rounding the number « $*$ »upwards)

$$
A_{k}=B_{k-1}^{\perp} A_{k-1} B_{k-1}^{\perp+}, B_{k}=B_{k-1}^{\perp} A_{k-1} B_{k-1}, C_{k}=C_{k-1} A_{k-1} B_{k-1}^{\perp+} .
$$

Equations (7), (8) for a set of indices $k=\overline{0, M}$ involve the matrices with the following properties:

$$
\begin{aligned}
& \left(B_{k} \mid B_{k}^{\perp+}\right)^{-1}=\left(\frac{B_{k}^{+}}{B_{k}^{\perp}}\right), \quad B_{k}^{\perp} B_{k}=0, \quad B_{k}^{+} B_{k}=I_{r}, \\
& \left(\frac{C_{k}}{C_{k}^{\perp}}\right)^{-1}=\left(C_{k}^{+} \mid C_{k}^{\perp+}\right), \quad C_{k} C_{k}^{\perp}=0, \quad C_{k} C_{k}^{+}=I_{m},
\end{aligned}
$$

Where the superscript « $\perp$ » denotes orthogonal annihilators (divisors of zero), and the superscript «+» denotes the Moore-Penrose pseudo inverse matrices. ${ }^{13,16-19}$ Also, we consider the recurrence formulae of controllers for the spectrum control on the corresponding decomposition levels, written down in reverse order:

- $M$-th decomposition level

$$
F_{M}=\left(\Phi_{M} B_{M}^{+}-B_{M}^{+} A_{M}\right) C_{M}^{+}
$$

${ }^{k}$-th decomposition level $(k=\overline{0, M-1})$

$$
F_{k}=\left(\Phi_{k} B_{k}^{-}-B_{k}^{-} A_{k}\right) C_{k}^{+}, B_{k}^{-}=B_{k}^{+}-F_{k+1} C_{k+1} B_{k}^{\perp}
$$

The multilevel decomposition procedure considered is then implemented.

\section{Algorithm for synthesis of the MIMO-system output control}

The following statement is true that has been proven ${ }^{13}$ and given here with an allowance for replacement of matrix annihilators, which are not possessed of a property of orthogonality, i.e., replacement with matrices, for which, in general, not necessarily the equality $B_{k}^{\perp} B_{k}^{\perp+}=I_{n-r}$ is implemented.

Theorem1. Let $m \geq r$, and the following matrices exist and are pair wise completely controllable:

$$
G_{k}^{\mathrm{T}}=B_{k}^{-} A_{k} C_{k}^{\perp}\left(B_{k}^{-} C_{k}^{\perp}\right)^{+}, H_{k}^{\mathrm{T}}=\left(B_{k}^{-} C_{k}^{\perp}\right)^{\perp}, k=\overline{0, M} \text {, Then, there exists a }
$$

nonempty set of matrices $K \quad i=\overline{0, M}$, such that

$$
\Phi_{i}=G_{i}+K_{i}^{\mathrm{T}} H_{i}=\left(B_{i}^{-} A_{i} C_{i}^{\perp}\right)^{\dot{\perp}}\left(B_{i}^{-} C_{i}^{\perp}\right)^{+}+K_{i}^{\mathrm{T}}\left(B_{i}^{-} C_{i}^{\perp}\right)^{\perp},(11) \text { and(9), (10) }
$$
satisfy the equalities of spectra

$$
\operatorname{eig}\left(A_{k}+B_{k} F_{k} C_{k}\right)=\bigcup_{i=k}^{M} \operatorname{eig}\left(\Phi_{i}\right), \quad k=\overline{1, M},
$$$$
A_{0}=A, \quad B_{0}=B, \quad C_{0}=C,
$$

$\mathrm{A}_{\mathrm{k}}=\mathrm{C}_{\mathrm{k}-1}^{\wedge} \mathrm{A}_{\mathrm{k}-1} \mathrm{C}_{\mathrm{k}-1}^{\wedge+}, \mathrm{B}_{\mathrm{k}}=\mathrm{B}_{\mathrm{k}-1} \mathrm{~A}_{\mathrm{k}-1} \mathrm{C}_{\mathrm{k}-1}^{\wedge+}, \mathrm{C}_{\mathrm{k}}=\mathrm{C}_{\mathrm{k}-1} \mathrm{~A}_{\mathrm{k}-1} \mathrm{C}_{\mathrm{k}-1}^{\wedge+}, \quad k=\overline{1, N}$ moreover, the following matrices exist and are pair wise completely controllable:

$$
\mathrm{G}_{\mathrm{k}}=\left(\mathrm{B}_{\mathrm{k}} \mathrm{C}_{\mathrm{k}}^{+}\right)^{+} \mathrm{B}_{\mathrm{k}}^{\wedge} \mathrm{A}_{\mathrm{k}} \mathrm{C}_{\mathrm{k}}^{+}, \mathrm{H}_{\mathrm{k}}=\left(\mathrm{B}_{\mathrm{k}} \mathrm{C}_{\mathrm{k}}^{+}\right)^{\wedge}, \mathrm{k}=\overline{0, \mathrm{~N}} .
$$

Then, there exists a nonempty set of matrices $\mathrm{L}_{\mathrm{i}}, i=\overline{0, N}$, such that $\Psi_{i}=\mathrm{G}_{\mathrm{i}}+\mathrm{H}_{\mathrm{i}} \mathrm{L}_{\mathrm{i}}^{\mathrm{T}}=\left(\mathrm{B}_{\mathrm{i}}^{\wedge} \mathrm{C}_{\mathrm{i}}^{+}\right)^{+} \mathrm{B}_{\mathrm{i}}^{\wedge} \mathrm{A}_{\mathrm{i}} \mathrm{C}_{\mathrm{i}}^{+}+\left(\mathrm{B}_{\mathrm{i}}^{\wedge} \mathrm{C}_{\mathrm{i}}^{+}\right)^{\wedge} \mathrm{L}_{\mathrm{i}}^{\mathrm{T}}$ and, for $F_{N}=B_{M}^{+}\left(C_{M}^{+} \Psi_{M}^{-} A_{M} C_{M}^{+}\right) F_{k}=B_{k}^{+}\left(C_{k}^{+} \Psi_{k}^{-} A_{k} C_{k}^{+}\right)$ 


$$
\begin{aligned}
& C_{k}^{-}=C_{k}^{+}-C_{k}^{\wedge T} B \underset{K+1}{F}{ }_{K+1}, \mathrm{k}=\overline{1, \mathrm{~N}-1} \text { it holds that } \\
& \text {, } \operatorname{eig}\left(\mathrm{A}_{\mathrm{k}}+\mathrm{B}_{\mathrm{k}} \mathrm{F}_{\mathrm{k}} \mathrm{C}_{\mathrm{k}}\right)=\bigcup_{\mathrm{i}=\mathrm{k}}^{\mathrm{N}} \operatorname{eig}\left(\Psi_{\mathrm{i}}\right), \mathrm{k}=\overline{1, \mathrm{~N}} \\
& \operatorname{eig}\left(\mathrm{A}_{0}+\mathrm{B}_{0} \mathrm{~F}_{0} \mathrm{C}_{0}\right)=\bigcup_{\mathrm{i}=0}^{\mathrm{N}} \operatorname{eig}\left(\Psi_{\mathrm{i}}\right)=\Lambda . \text { It should be noted that }
\end{aligned}
$$

here, as in the algorithms described, ${ }^{13,16-19}$ only semi orthogonal and pseudo inverse matrices are used in the transformations, which at least do not reduce the condition number of the equations. Moreover, this approach does not impose restrictions in the form of the differentiation between the algebraic and geometric multiplicities of the elements of the spectrum to be assigned; there are also no restrictions on the size of the problem. This is confirmed by simulation, which shows a high relative accuracy of spectrum control and the practical absence of restrictions on the size of system (3).

\section{Analytical synthesis of a single-airscrew helicopter's longitudinal motion control}

In accordance with the problem statement, it is required to find explicitly a formula of controller $\boldsymbol{F}$ in the control law (5) that can be expressed in this case as:

$$
\left(\begin{array}{c}
u_{x} \\
u_{g p}
\end{array}\right)=F C\left(\begin{array}{c}
v_{x} \\
v_{y} \\
\omega_{z} \\
v
\end{array}\right)=F\left(\begin{array}{c}
v_{x} \\
\omega_{z} \\
v
\end{array}\right),
$$

And provides for the close-loop system «HS + control system» of a specified earlier spectrum(6). We perform for the system (2) with matrices(3), (4) - the multilevel decomposition described in Section 1 , which has in this case two $\operatorname{eig}\left(\mathrm{A}_{0}+\mathrm{B}_{0} \mathrm{~F}_{0} \mathrm{C}_{0}\right)=\bigcup \bigcup_{i=0} \operatorname{eig}\left(\Phi_{\mathrm{i}}\right)=\Lambda$. The condition $m \geq r$ in Theorem 1 is not restrictive; it is introduced to indicate that, in the present case $F$, matrix from (5) is conventionally considered as a matrix of controller (i.e. the number of inputs is less than the number of outputs). For the case $m \leq r$, Theorem 1 has a dual formulation, and matrix $F$ is replaced with the observer matrix $L$ (the number of inputs is greater than the number of outputs).

Theorem 2. Let $m \leq r, \quad N=\operatorname{ceil}(n / m)$, and the following decomposition of system (3) hold: decomposition levels ( $M=1$ ): zero level (7) and first level (8). Therefore, we will have

$$
\begin{aligned}
& B_{0}^{\perp}=\left(\begin{array}{cccc}
l_{11} & l_{11} & 1 & 0 \\
0 & 0 & 0 & 1
\end{array}\right), B_{0}^{\perp+}=\left(\begin{array}{cc}
l_{11} l^{-1} & 0 \\
l_{12} l^{-1} & 0 \\
l^{-1} & 0 \\
0 & 1
\end{array}\right), \\
& \mathrm{A}_{1}=\left(\begin{array}{cc}
a_{11}^{1} & a_{12}^{1} \\
a_{21}^{1} & 0
\end{array}\right), \mathrm{B}_{1}=\left(\begin{array}{ll}
b_{11}^{1} & b_{12}^{1} \\
b_{21}^{1} & b_{22}^{1}
\end{array}\right), \mathrm{C}_{1}=\left(\begin{array}{cc}
c_{11}^{1} & c_{12}^{1} \\
c_{21}^{1} & c_{22}^{1} \\
c_{31}^{1} & 0
\end{array}\right),
\end{aligned}
$$

Where, taking in view the following notation $l^{*}=b_{11} b_{22}-b_{12} b_{21}$, the matrices' elements in(12), (13) is equal to:

$$
l_{11}=\frac{b_{21} b_{32}-b_{22} b_{31}}{l^{*}}, l_{12}=\frac{-\left(b_{11} b_{32}-b_{12} b_{31}\right)}{l^{*}}, \quad l=l_{11}^{2}+l_{12}^{2}+1,
$$

$$
\begin{aligned}
& a_{11}^{1} l=a_{33}+a_{13} l_{11}+a_{23} l_{12}+a_{31} l_{11}+a_{32} l_{12}+a_{11} l_{11}^{2}+ \\
& +a_{22} l_{12}^{2}+a_{12} l_{11} l_{12}+a_{21} l_{11} l_{12}, \\
& a_{12}^{1}=a_{13} l_{11}+a_{24} l_{12}, a_{21}^{1}=l^{-1}, \quad b_{21}^{1}=b_{31}, b_{22}^{1}=b_{32} \text {, } \\
& b_{11}^{1}=b_{11}\left(a_{31}+a_{11} l_{11}+a_{21} l_{12}\right)+b_{21}\left(a_{32}+a_{12} l_{11}+a_{22} l_{12}\right)+ \\
& +b_{31}\left(a_{33}+a_{13} l_{11}+a_{23} l_{12}\right) \text {, } \\
& b_{12}^{1}=b_{12}\left(a_{31}+a_{11} l_{11}+a_{21} l_{12}\right)+b_{22}\left(a_{32}+a_{12} l_{11}+a_{22} l_{12}\right)+ \\
& +b_{32}\left(a_{33}+a_{13} l_{11}+a_{23} l_{12}\right) \text {, } \\
& c_{11}^{1}=\left(a_{13}+a_{11} l_{11}+a_{12} l_{12}\right) l^{-1}, \\
& c_{12}^{1}=a_{13}, c_{21}^{1}=\left(a_{33}+a_{31} l_{11}+a_{32} l_{12}\right) l^{-1}, c_{22}^{1}=0, c_{31}^{1}=l^{-1} \text {. }
\end{aligned}
$$

To check the controllability conditions in Theorem 1, we calculate the matrices:

$$
C_{0}^{\perp \mathrm{T}}=\left(\begin{array}{llll}
0 & 1 & 0 & 0
\end{array}\right), B_{0}^{+}=\left(\begin{array}{llll}
b_{11}^{0+} & b_{12}^{0+} & b_{14}^{0+} & 0 \\
b_{21}^{0+} & b_{22}^{0+} & b_{23}^{0+} & 0
\end{array}\right), \quad \text { in which, in }
$$
presence of the introduced parameter

$$
\begin{gathered}
b^{0+}=b_{11}^{2} b_{22}^{2}+b_{11}^{2} b_{32}^{2}-2 b_{11} b_{12} b_{21} b_{22}-2 b_{11} b_{12} b_{31} b_{32}+ \\
+b_{12}^{2} b_{21}^{2}+b_{12}^{2} b_{31}^{2}+b_{21}^{2} b_{32}^{2}-2 b_{21} b_{22} b_{31} b_{32}+b_{22}^{2} b_{31}^{2},
\end{gathered}
$$

Corresponding elements can be expressed as

$$
\begin{aligned}
& b_{11}^{0+}=\frac{b_{11} b_{22}^{2}-b_{12} b_{21} b_{22}+b_{11} b_{32}^{2}-b_{12} b_{31} b_{32}}{b^{0+}}, \\
& b_{12}^{0+}=\frac{b_{21} b_{12}^{2}-b_{11} b_{22} b_{12}+b_{21} b_{32}^{2}-b_{22} b_{31} b_{32}}{b^{0+}}, \\
& b_{13}^{0+}=\frac{b_{31} b_{12}^{2}-b_{11} b_{32} b_{12}+b_{31} b_{22}^{2}-b_{21} b_{32} b_{22}}{b^{0+}}, \\
& b_{21}^{0+}=\frac{b_{12} b_{21}^{2}-b_{11} b_{22} b_{21}+b_{12} b_{31}^{2}-b_{11} b_{32} b_{31}}{b^{0+}}, \\
& b_{22}^{0+}=\frac{b_{22} b_{11}^{2}-b_{12} b_{21} b_{11}+b_{22} b_{31}^{2}-b_{21} b_{32} b_{31}}{b^{0+}}, \\
& b_{23}^{0+}=\frac{b_{32} b_{11}^{2}-b_{12} b_{31} b_{11}+b_{32} b_{21}^{2}-b_{22} b_{31} b_{21}}{b^{0+}} . \\
& b_{22}^{0+}=\frac{b_{22} b_{11}^{2}-b_{12} b_{21} b_{11}+b_{22} b_{31}^{2}-b_{21} b_{32} b_{31}}{b^{0+}}, \\
& b_{23}^{0+}=\frac{b_{32} b_{11}^{2}-b_{12} b_{31} b_{11}+b_{32} b_{21}^{2}-b_{22} b_{31} b_{21}}{b^{0+}} . \\
& n^{0+}
\end{aligned}
$$

Besides, the following equations take place:

$$
\begin{aligned}
& H_{0}=\left(B_{0}^{+} C_{0}^{\perp}\right)^{\perp \mathrm{T}}=\left(-\frac{b_{22}^{0+}}{b_{12}^{0+}} 1\right)^{\mathrm{T}}, \\
& G_{0}=\left(B_{0}^{-} C_{0}^{\perp}\right)^{+\mathrm{T}}\left(B_{0}^{-} A_{0} C_{0}^{\perp}\right)^{\mathrm{T}}= \\
& =\left(\begin{array}{ll}
\frac{b_{12}^{0+}\left(a_{12} b_{11}^{0+}+a_{22} b_{12}^{0+}+a_{32} b_{13}^{0+}\right)}{\left(b_{12}^{0+}\right)^{2}+\left(b_{22}^{0+}\right)^{2}} & \frac{b_{12}^{0+}\left(a_{12} b_{21}^{0+}+a_{22} b_{22}^{0+}+a_{32} b_{23}^{0+}\right)}{\left(b_{12}^{0+}\right)^{2}+\left(b_{22}^{0+}\right)^{2}} \\
\frac{b_{22}^{0+}\left(a_{12} b_{11}^{0+}+a_{22} b_{12}^{0+}+a_{32} b_{13}^{0+}\right)}{\left(b_{12}^{0+}\right)^{2}+\left(b_{22}^{0+}\right)^{2}} & \frac{b_{22}^{0+}\left(a_{12} b_{21}^{0+}+a_{22} b_{22}^{0+}+a_{32} b_{23}^{0+}\right)}{\left(b_{12}^{0+}\right)^{2}+\left(b_{22}^{0+}\right)^{2}}
\end{array}\right)
\end{aligned}
$$




$$
\begin{gathered}
C_{1}^{\perp \mathrm{T}}=0, \quad B_{1}^{+}=\left(\begin{array}{ll}
b_{11}^{1+} & b_{12}^{1+} \\
b_{21}^{1+} & b_{22}^{1+}
\end{array}\right), \\
H_{1}=\left(B_{1}^{+} C_{1}^{\perp \mathrm{T}}\right)^{\perp \mathrm{T}}=I_{2}, H_{1}^{+}=\left(H_{1}\right)^{+}=I_{2}, \\
G_{1}=\left(B_{1}^{+} C_{1}^{\perp \mathrm{T}}\right)^{+\mathrm{T}}\left(B_{1}^{+} A_{1} C_{1}^{\perp \mathrm{T}}\right)^{\mathrm{T}}=0,
\end{gathered}
$$

Where, for brevity, the following notation is used:

$$
h_{1}^{+}=b_{11}^{1} b_{22}^{1}-b_{12}^{1} b_{21}^{1}
$$

$b_{11}^{1+}=b_{22}^{1} / h_{1}^{+}, b_{12}^{1+}=-b_{12}^{1} / h_{1}^{+}, b_{21}^{1+}=-b_{21}^{1} / h_{1}^{+}, b_{22}^{1+}=b_{11}^{1} / h_{1}^{+}$. For the zero and first decomposition levels, we calculate the ranks of the following block matrices $\left(H_{1}: G_{1} H_{1}\right),\left(H_{1}: G_{1} H_{1}\right)$ as a result we will obtain: $\operatorname{rank}\left(H_{1} \mid G_{1} H_{1}\right)=2, \quad \operatorname{rank}\left(H_{1} \mid G_{1} H_{1}\right)=2$, this corresponds to the number of "independent" inputs $r=2$. Therefore, each level of decomposition satisfies the controllability condition in Theorem 1. According to the form of controllers(9), (10) we define a matrix whose Eigen values will be assigned to the first decomposition level. Since matrices (15) are invertible, we may choose any matrix Ô that has the prescribed spectrum for the first decomposition level. For simplicity, we set this matrix diagonal $\hat{O}_{1}=\operatorname{diag}\left(\hat{\lambda}_{1}, \hat{\lambda}_{2}\right)$, and calculate the pseudo inverse matrix

$$
C_{1}^{+}=\left(\begin{array}{lll}
c_{11}^{1+} & c_{12}^{1+} & c_{13}^{1+} \\
c_{21}^{1+} & c_{22}^{1+} & c_{23}^{1+}
\end{array}\right) .
$$

Here, again for brevity, we introduce the notation

$$
\begin{aligned}
& c^{1+}=\left(c_{11}^{1} c_{22}^{1}\right)^{2}-2 c_{11}^{1} c_{12}^{1} c_{21}^{1} c_{22}^{1}+\left(c_{12}^{1} c_{21}^{1}\right)^{2}+\left(c_{12}^{1} c_{31}^{1}\right)^{2}+\left(c_{22}^{1} c_{31}^{1}\right)^{2}, \\
& c_{11}^{1+}=c_{22}^{1}\left(c_{11}^{1} c_{22}^{1}-c_{12}^{1} c_{21}^{1}\right) / c^{1+}, \quad c_{12}^{1+}=-c_{12}^{1}\left(c_{11}^{1} c_{22}^{1}-c_{12}^{1} c_{21}^{1}\right) / c^{1+}, \\
& c_{13}^{1+}=c_{31}^{1}\left(c_{12}^{1} c_{12}^{1}+c_{22}^{1} c_{22}^{1}\right) / c^{1+}, \quad c_{21}^{1+}=\left(c_{12}^{1} c_{21}^{1} c_{21}^{1}-c_{11}^{1} c_{21}^{1} c_{22}^{1}+c_{12}^{1} c_{31}^{1} c_{31}^{1}\right) / c^{1+}, \\
& c_{22}^{1+}=\left(c_{22}^{1} c_{11}^{1} c_{11}^{1}-c_{12}^{1} c_{21}^{1} c_{11}^{1}+c_{22}^{1} c_{31}^{1} c_{31}^{1}\right) / c^{1+}, \\
& c_{23}^{1+}=-c_{31}^{1}\left(c_{11}^{1} c_{12}^{1}-c_{21}^{1} c_{22}^{1}\right) / c^{1+} .
\end{aligned}
$$

Based on equation(9), the first decomposition level yields the following formula for the controller:

Where the following notation is used:

$$
F_{1}=B_{1}^{+}\left(\Phi_{1}-A_{1}\right) C_{1}^{+}=\left(\begin{array}{ccc}
f_{11}^{1} & f_{12}^{1} & f_{13}^{1} \\
f_{21}^{1} & f_{22}^{1} & f_{23}^{1}
\end{array}\right),
$$

$$
\begin{aligned}
& d_{1}=a_{12}^{1} b_{11}^{1+}-b_{12}^{1+} \hat{\lambda}_{1}, d_{2}=a_{11}^{1} b_{11}^{1+}+a_{21}^{1} b_{12}^{1+}-b_{11}^{1+} \hat{\lambda}_{1}, \\
& d_{3}=a_{12}^{1} b_{21}^{1+}-b_{22}^{1+} \hat{\lambda}_{2}, d_{4}=a_{11}^{1} b_{21}^{1+}+a_{21}^{1} b_{22}^{1+}-b_{11}^{1+} \hat{\lambda}_{2},
\end{aligned}
$$

and where the components are:

$$
f_{11}^{1}=-c_{21}^{1+} d_{1}-c_{11}^{1+} d_{2}, f_{12}^{1}=-c_{22}^{1+} d_{1}-c_{12}^{1+} d_{2}, f_{13}^{1}=-c_{23}^{1+} d_{1}-c_{13}^{1+} d_{2},
$$

To calculate matrix $B_{0}^{-}$that is needed for determining the zero level controller, we use the second formula in(10). As a result, we obtain the expression

$$
\begin{gathered}
B^{-} \\
0
\end{gathered}=B^{+}-F C B^{\perp}=\left(\begin{array}{ccccc}
b_{11}^{m} & b_{12}^{m} & b_{13}^{m} & b_{14}^{m} \\
b_{21}^{m} & b_{22}^{m} & b_{23}^{m} & b_{24}^{m}
\end{array}\right) .
$$

Here we denote linear combinations:

$$
\begin{aligned}
& b_{11}^{m}=b_{11}^{0+}-l_{11}\left(c_{11}^{1} f_{11}^{1}+c_{21}^{1} f_{12}^{1}+c_{31}^{1} f_{13}^{1}\right), \\
& b_{12}^{m}=b_{12}^{0+}-l_{12}\left(c_{11}^{1} f_{11}^{1}+c_{21}^{1} f_{12}^{1}+c_{31}^{1} f_{13}^{1}\right), \\
& b_{13}^{m}=b_{13}^{0+}-c_{11}^{1} f_{11}^{1}-c_{21}^{1} f_{12}^{1}-c_{31}^{1} f_{13}^{1}, \\
& b_{14}^{m}=-c_{12}^{1} f_{11}^{1}-c_{22}^{1} f_{12}^{1}, \\
& b_{21}^{m}=b_{21}^{0+}-l_{11}\left(c_{11}^{1} f_{21}^{1}+c_{21}^{1} f_{22}^{1}+c_{31}^{1} f_{23}^{1}\right), \\
& b_{22}^{m}=b_{22}^{0+}-l_{12}\left(c_{11}^{1} f_{21}^{1}+c_{21}^{1} f_{22}^{1}+c_{31}^{1} f_{23}^{1}\right), \\
& b_{23}^{m}=b_{23}^{0+}-c_{11}^{1} f_{21}^{1}-c_{21}^{1} f_{22}^{1}-c_{31}^{1} f_{23}^{1}, \\
& b_{24}^{m}=-c_{12}^{1} f_{21}^{1}-c_{22}^{1} f_{22}^{1} .
\end{aligned}
$$

According to Theorem 1, we complete the system of the zero decomposition level using (7). For this purpose we use equation (14) and, applying new notation, we obtain

$$
\begin{aligned}
& \text { Where we denote } \\
& \qquad \begin{array}{l}
a_{11}^{a}=b_{12}^{0+} d_{1} / d, a_{12}^{a}=b_{12}^{0+} d_{2} / d, \\
a_{21}^{a}=b_{22}^{0+} d_{1} / d, a_{22}^{a}=b_{22}^{0+} d_{2} / d, \\
d=\left(b_{12}^{0+}\right)^{2}+\left(b_{22}^{0+}\right)^{2}, d_{1}=a_{12} b_{11}^{m}+a_{22} b_{12}^{m}+a_{32} b_{13}^{m}, \\
d_{2}=a_{12} b_{21}^{m}+a_{22} b_{22}^{m}+a_{32} b_{23}^{m} .
\end{array}
\end{aligned}
$$$$
G_{0}=\left(\begin{array}{ll}
a_{11}^{a} & a_{12}^{a} \\
a_{21}^{a} & a_{22}^{a}
\end{array}\right)
$$

Now, we should determine $\mathrm{O}$ for the zero decomposition level. For this purpose, we decompose ${ }^{0}$ the matrices $H_{0}, G_{0}$ of the zero level into two sublevels and calculate the corresponding matrices. We obtain

$$
\begin{aligned}
& \left(H_{0}\right)_{0}=H_{0},\left(G_{0}\right)_{0}=G_{0},\left(H_{0}\right)_{0}^{\perp}=\left(\begin{array}{ll}
b_{12}^{m} / b_{22}^{m} & 1
\end{array}\right), \\
& \left(H_{0}\right)_{0}^{\perp+}=\left(\begin{array}{l}
b_{12}^{m} b_{22}^{m} / b^{m} \\
\left(b_{12}^{m}\right)^{2} / b^{m}
\end{array}\right), \\
& \left(H_{0}\right)_{0}^{+}=\left(\begin{array}{ll}
-b_{12}^{m} b_{22}^{m} / b^{m} & \left.\left(b_{12}^{m}\right)^{2} / b^{m}\right) .
\end{array}\right.
\end{aligned}
$$

Here $b^{m}=\left(b_{12}^{m}\right)^{2}+\left(b_{22}^{m}\right)^{2}$, and, in addition,

$$
\begin{aligned}
& \left(G_{0}\right)_{1}=\left(H_{0}\right)_{0} G_{0}\left(H_{0}\right)_{0}^{\perp}=a_{1}^{a a}= \\
& =\left(b_{22}^{m}\right)^{2}\left(a_{22}^{a}+a_{12}^{a} b_{12}^{m} / b_{22}^{m}\right) / b^{m}+ \\
& +b_{12}^{m} b_{22}^{m}\left(a_{21}^{a}+a_{11}^{a} b_{12}^{m} / b_{22}^{m}\right) / b^{m}, \\
& \left(H_{0}\right)_{1}=\left(H_{0}\right)_{0}^{\perp} G_{0} H_{0}=b_{11}^{b b} .
\end{aligned}
$$

Besides,

$b_{11}^{b b}=a_{22}^{a}-\left(b_{22}^{m}\left(a_{21}^{a}+a_{11}^{a} b_{12}^{m} / b_{22}^{m}\right) / b_{12}^{m}+a_{12}^{a} b_{12}^{m} / b_{22}^{m}\right.$. (16)

Using the values of ${ }_{\left(H_{0}\right)=b_{0} \mid}(16)$, we then find the matrix (scalar, in this case) $\left(H_{0}\right)_{1}^{+}=b_{p 11}$, where $b_{p 11}=1 / b_{11}^{b b}$.

Let us now assign the Eigen value as a scalar matrix $\left(\hat{\mathrm{O}}_{0}\right)_{1}=\hat{\lambda}_{3}$ and calculate the matrix of feedback coefficients for the first sublevel of the zero decomposition level. We obtain $k_{1}=-b_{p 11}\left(a_{1}^{a a}-\widehat{\lambda}_{3}\right)$. Next, we calculate the matrix

$$
h_{11}^{0-}=b_{p 11} b_{12}^{m}\left(\left(a_{1}^{a a}-\hat{\lambda}_{3}\right) / b_{22}^{m}+b_{12}^{m} b_{22}^{m} / b^{m},\right.
$$




\section{Where}

$h_{11}^{0-}=b_{p 11} b_{12}^{m}\left(\left(a_{1}^{a a}-\hat{\lambda}_{3}\right) / b_{22}^{m}+b_{12}^{m} b_{22}^{m} / b^{m}\right.$,

$h_{12}^{0-}=b_{p 11}\left(a_{1}^{a a}-\hat{\lambda}_{3}\right)+\left(b_{12}^{m}\right)^{2} / b^{m}$, and specify again the matrix of Eigen values of the zero sublevel of the zero decomposition level by $\left(\Phi_{0}\right)_{0}=\bar{\lambda}$.

Finally, we find matrix $K_{0}$ by the rule

$$
K_{0}=\left(\Phi_{0}\right)_{0}\left(H_{0}\right)_{0}^{-}-\left(H_{0}\right)_{0}^{-} G_{0}=\left(\begin{array}{ll}
K_{11} & K_{12}
\end{array}\right),
$$

Where in the case under examination

$$
\begin{aligned}
& K_{11}=a_{11}^{a}\left(\left(b_{12}^{m} b_{22}^{m} / b^{m}-b_{p 11} b_{12}^{m}\left(a_{1}^{a a}-\hat{\lambda}_{3}\right) / b_{22}^{m}\right)-a_{21}^{a}\left(b_{p 11}\left(a_{1}^{a a}-\hat{\lambda}_{3}\right)\right.\right. \\
& \left.\quad+\left(b_{12}^{m}\right)^{2} / b^{m}\right)-\hat{\lambda}_{4}\left(b_{12}^{m} b_{22}^{m} / b^{m}-b_{p 11} b_{12}^{m}\left(a_{1}^{a a}-\hat{\lambda}_{3}\right) / b_{22}^{m}\right), \\
& K_{12}=\hat{\lambda}_{4}\left(b_{p 11}\left(a_{1}^{a a}-\hat{\lambda}_{3}\right)+\left(b_{12}^{m}\right)^{2} / b^{m}\right)-a_{22}^{a}\left(b_{p 11}\left(a_{1}^{a a}-\hat{\lambda}_{3}\right)+\right. \\
& \left.+\left(b_{12}^{m}\right)^{2} / b^{m}\right)+a_{12}^{a}\left(\left(b_{12}^{m} b_{22}^{m} / b^{m}-b_{p 11} b_{12}^{m}\left(a_{1}^{a a}-\hat{\lambda}_{3}\right) / b_{22}^{m}\right) .\right.
\end{aligned}
$$

As a result, we obtain using equation(11), the matrix $\hat{O}_{0}$, whose eigen values $\hat{\lambda}_{3}, \hat{\lambda}_{4}$, are ensured by the output controller, namely,

$$
\Phi_{0}=\left(\begin{array}{cc}
a_{11}^{a}-\frac{b_{22}^{m}}{b_{12}^{m}} K_{11} & a_{21}^{a}+K_{11} \\
a_{12}^{a}-\frac{b_{22}^{m}}{b_{12}^{m}} K_{12} & a_{22}^{a}+K_{12}
\end{array}\right) .
$$

Further calculations, which were described, for instance, ${ }^{13,17-19}$ finally yield the following formula for the SH output controller vector:

$$
F=\left(\begin{array}{lll}
f_{11} & f_{12} & f_{13} \\
f_{21} & f_{22} & f_{23}
\end{array}\right)
$$

Here elements of the following notation:

$$
d_{21}=a_{12}^{a}-\frac{b_{22}^{m}}{b_{12}^{m}} K_{12}, d_{22}=a_{22}^{a}+K_{12}, d_{21}=a_{12}^{a}-\frac{b_{22}^{m}}{b_{12}^{m}} K_{12}, d_{22}=a_{22}^{a}+K_{12},
$$

Can be expressed as

$$
\begin{aligned}
& f_{11}=b_{11}^{m} d_{11}-b_{12}^{m} a_{21}-b_{13}^{m} a_{31}-b_{11}^{m} a_{11}+b_{21}^{m} d_{12}, \\
& f_{12}=b_{13}^{m} d_{11}-b_{13}^{m} a_{33}-b_{14}^{m}+b_{23}^{m} d_{12}, \\
& f_{13}=b_{14}^{m} d_{11}-b_{13}^{m} a_{34}-b_{11}^{m} a_{13}-b_{12}^{m} a_{24}+b_{24}^{m} d_{12}, \\
& f_{21}=b_{11}^{m} d_{21}-b_{22}^{m} a_{21}-b_{23}^{m} a_{31}-b_{21}^{m} a_{11}+b_{21}^{m} d_{22}, \\
& f_{22}=b_{13}^{m} d_{21}-b_{23}^{m} a_{33}-b_{24}^{m}+b_{23}^{m} d_{22}, \\
& f_{23}=b_{14}^{m} d_{21}-b_{23}^{m} a_{34}-b_{22}^{m} a_{24}-b_{21}^{m} a_{13}+b_{24}^{m} d_{22} .
\end{aligned}
$$

The synthesized controller (the SH control system laws) ensures exactly the specified spectrum (6) for controlled longitudinal motion of the helicopter. This assertion can be directly checked with the help of appropriate analytical calculations. For this purpose it is sufficient to make use of the package Symbolic Toolbox Matlab; namely, one can use the eig instruction to calculate the eigen values of the $\boldsymbol{A}+\boldsymbol{B F C}$ matrix, where matrices can be expressed as(4), and the $\boldsymbol{F}$ matrix is determined by (17)

\section{Numerical analysis}

Suppose that we want the closed-loop system "SH + control system" to have the following multiple real spectrum

$$
\Lambda=\{-1.5,-1.5,-1.5,-1.5\}
$$

Let use for the hypothetical SH the $\boldsymbol{A}, \boldsymbol{B}$ and $\boldsymbol{C}$ (4) matrices having the following numerical values:

$$
\begin{aligned}
A & =\left(\begin{array}{cccc}
-0.0598 & -0.0233 & 0.1095 & -0.1703 \\
-0.0268 & -0.8899 & -1.2091 & -0.0135 \\
1.5158 & -2.3207 & -2.3464 & 0 \\
0 & 0 & 1 & 0
\end{array}\right), \\
B & =\left(\begin{array}{cc}
-0.3050 & -0.0228 \\
-1.2817 & 2.0890 \\
28.178 & 22.7255 \\
0 & 0
\end{array}\right)
\end{aligned}
$$

With use of equation (17) for the controller we obtain the numerical value for the matrix $F$. As a result we have

$$
F=\left(\begin{array}{ccc}
3.6163 & -1.8622 & -3.4996 \\
-2.7936 & 2.2309 & 3.6237
\end{array}\right) \text {. }
$$

The computation of Eigen values of the matrix A + BFC yields the set

$\operatorname{eig}(A+B F C)=\{-1.5,-1.5151,-1.4924 \pm 0.0131 i\}, \quad$ Which lies quite close to the specified values (18). Note that to implement the sets (18) using the method ${ }^{5}$ is not possible. ${ }^{20,21}$

\section{Conclusion}

The problem of a hypothetical single-airscrew helicopter's longitudinal motion stabilization for lack of information about the vertical speed of its motion has been analytically solved. The solution is based on the method of the output signal control synthesis that provides a specified spectrum of the MIMO-system's motion, presented ${ }^{13,16-19}$.

\section{Acknowledgements}

My Institute's (Bauman Moscow State Technical University) representative is fully aware of this submission.

\section{Conflict of interest}

The author declares there is no conflict of interest.

\section{References}

1. Kim FD, Celi R. Simulation Modeling for Combat Rotorcraft Flight Control Design. Joint Research Interchange. NCA 2-310. Univ Maryland; 1990.

2. Miao WL, Huber HB. Rotor Aero elastic Stability Coupled with Helicopter Body Motion. NASA SP-352. 1974

3. Tischler MB. Assessment of digital flight-control technology for advanced combat rotorcraft. J Amer Helicopter Soc. 1989;34(4):66-76.

4. Han Z. Eigenstructure assignment using dynamical compensator. Int $J$ Control. 1989;49(1):233-245.

5. Kautsky J, Nichols NK, Van Dooren P. Robust pole assignment in linear state feedback. Int J Control. 1985;41(5):1129-1155.

6. Yang K, Orsi R. Static Output Feedback via a Trust Region Approach. IEEE Trans Automat Control. 2007:2146-2150. 
7. Quadrat A. On a generalization of the Youla-Kucera parametrization. Part I: The fractional ideal approach to SISO systems. Syst. Control Lett. 2003;50(2):135-148.

8. Krasovskii AA, Vavilov Yu A, Suchkov AI. Automatic control systems of the aircrafts. Moscow: The Air Force Engineering Academy. Russia.

9. Youla DC, Jabr HA, Bongiorno JJ. Modern Wiener-Hopf design of optimal controllers. Pt 2 Multivariable case. IEEE Trans Automat Control. 1976;21(3):319-338.

10. Mori K. Parametrization of the stabilizing controllers over a commutative ring with applications to multidimensional systems. IEEE Trans Circ Sys. 2002;49(6):743-752.

11. Kucera V. Discrete linear control: the polynomial equation approach. New York: Wiley; 1979.

12. Manousiouthakis $\mathrm{V}$, On the parametrization of all stabilizing decentralized controllers. Systems and Control Letters. 1993;21(5):397-403.

13. Zubov NE, Zybin E Yu, Mikrin EA, et al. Output control of a spacecraft motion spectrum. Comput Syst Sci Int. 2014;53(4):576-586.

14. Mikrin EA, Zubov NE, Misrikhanov MSh, et al. Invariant control of a single-airscrew helicopter's low-altitude flight. The Automation. Up-todate Technologies. 2015;6:3-8.
15. Leonov GA, Shumafov MM. Methods of the linear controlled systems stabilization. Saint-Petersburg: Saint-Petersburg State University Publishing House. 2005.

16. Misriknanov MSh, Ryabchenko VN. Pole placement in large dynamical systems with many inputs and outputs. Dokl Math. 2011;84:591-593.

17. Zubov N.EMikrin EA, Misrikhanov MSh, et al. Synthesis of decoupling laws for attitude stabilization of a spacecraft. Computer and Systems Sciences International. 2012;51(1):80-96.

18. Zubov NE, Mikrin EA, Misrikhanov MSh. et al. Modification of the exact pole placement method and its application for the control of spacecraft motion. Comput Syst Sci Int. 2013;52(2):279-292.

19. Zubov NE, Mikrin EA, Ryabchenko VN. Matrix methods in the theory and practice of aircraft automatic control systems. Moscow: Bauman State Technical University Publishing House. 2016.

20. Hautus MLJ, Heymann H. Linear feedback decoupling: transfer function analysis. IEEE Trans Automat Control. 1983;28(8):823-832.

21. Lin Ch An, Hsien T Fu. Decoupling controller design for linear multivariable plants. IEEE Trans Automat Control. 1991;36(4):485-489. 